\title{
Presence of Epstein-Barr virus in lymphoepithelioma-like carcinoma of the middle ear
}

Suet Y Leung, Siu T Yuen, Chiu M Ho, Wai K Kwong, Lap P Chung

\begin{abstract}
Aim-To examine the association of Epstein-Barr virus (EBV) with carcinoma of the ear.

Methods-Five non-keratinising squamous cell carcinomas and two undifferentiated carcinomas of the ear were examined. In situ hybridisation was used to localised EBV-encoded RNAs (EBER). Immunohistochemical methods to detect LMP-1 and EBNA2 were performed in the EBER positive cases.

Results-Two cases were EBER positive, including one non-keratinising and one undifferentiated carcinoma. Both showed identical morphology to those arising from the nasopharynx, with abundant lymphoid stroma. They were both negative for LMP-1 and EBNA2.

Conclusions-EBV associated carcinoma with the morphology of lymphoepithelioma can also arise from the middle ear. (F Clin Pathol 1998;51:602-605)
\end{abstract}

Keywords: Epstein-Barr virus; lymphoepithelioma; ear

The middle ear lining is in direct continuity with the nasopharynx through the Eustachian
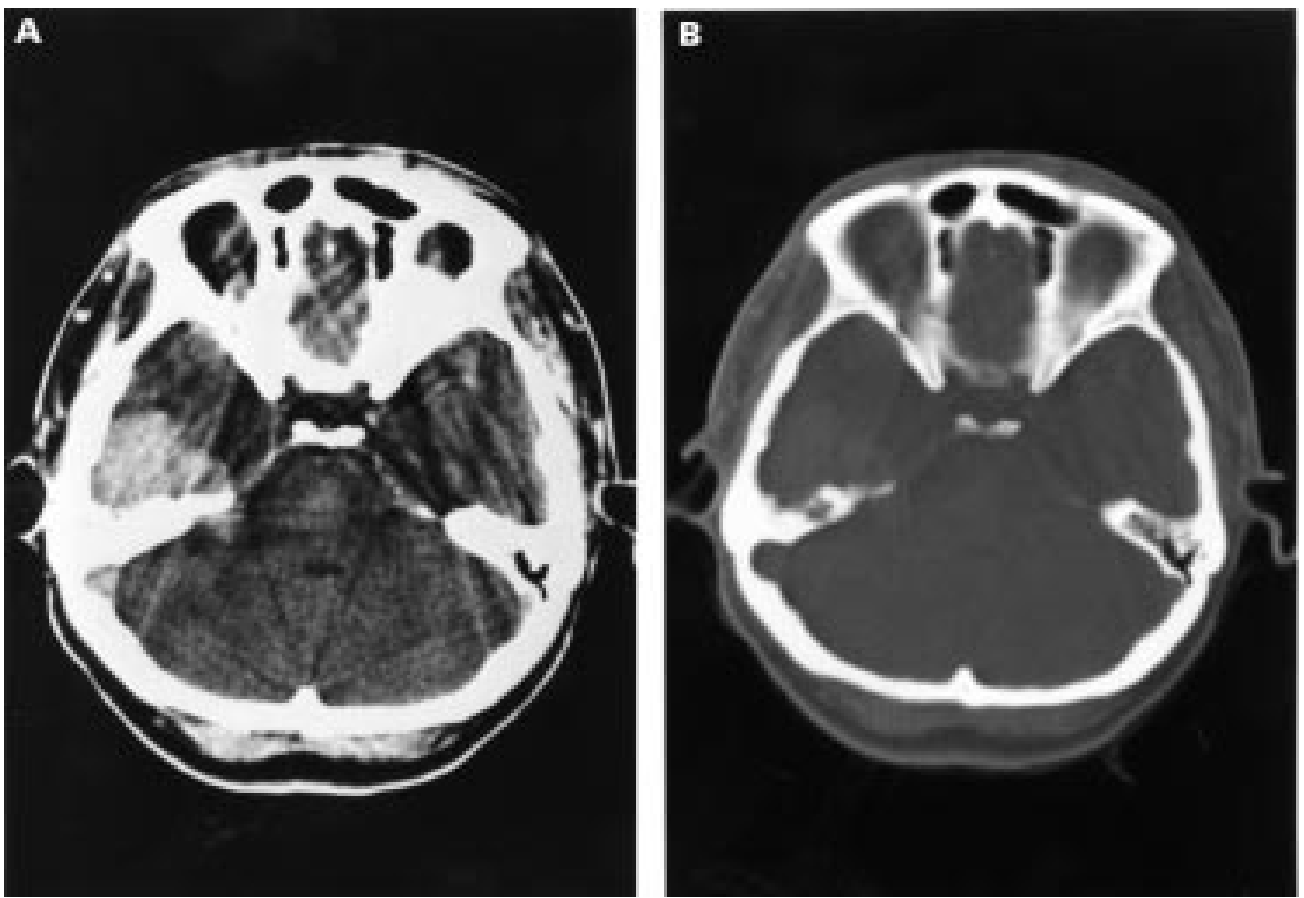

Figure 1 Case 1. (A) Non-contrast computed tomography shows the presence of a tumour arising from the petrous temporal bone with extension into the right middle cranial fossa. (B) An intraosseous component with destruction of the petrous temporal bone is seen in the bone window.

tube. Developmentally, both are derived from the second pharyngeal pouch. Although one of the most common sites for the development of nasopharyngeal carcinoma is in the nasopharyngeal cushion near the origin of the Eustachian tubes, lymphoepithelioma or EBV associated carcinoma has never been described in the middle or external ear canal. Keratinising squamous cell carcinoma is by far the most common cancer of the ear. EBV is mostly associated with non-keratinising anaplastic carcinomas, and keratinising squamous cell carcinomas are usually negative for EBV in other anatomical sites apart from the nasopharynx. This finding stimulated examination of a series of non-keratinising squamous cell carcinoma and undifferentiated carcinoma of the ear to look for the presence of EBV.

\section{Methods}

\section{MATERIALS}

Five non-keratinising squamous cell carcinoma and two undifferentiated carcinoma of the ear were included in this study. The cases were retrieved by reviewing the pathology records and histological sections, along with a review of the clinical records including the computerised tomography scans to exclude the involvement

The University of Hong Kong, Queen Mary Hospital, Hong Kong:

Department of

Pathology

S Y Leung

$S$ T Yuen

L P Chung

Department of

Surgery

$\mathrm{C} M \mathrm{Ho}$

Department of Radiation Oncology W K Kwong

Correspondence to: Dr Suet Yi Leung,

Department of Pathology, Queen Mary Hospital, The Hong Kong.

Accepted for publication 23 April 1998 

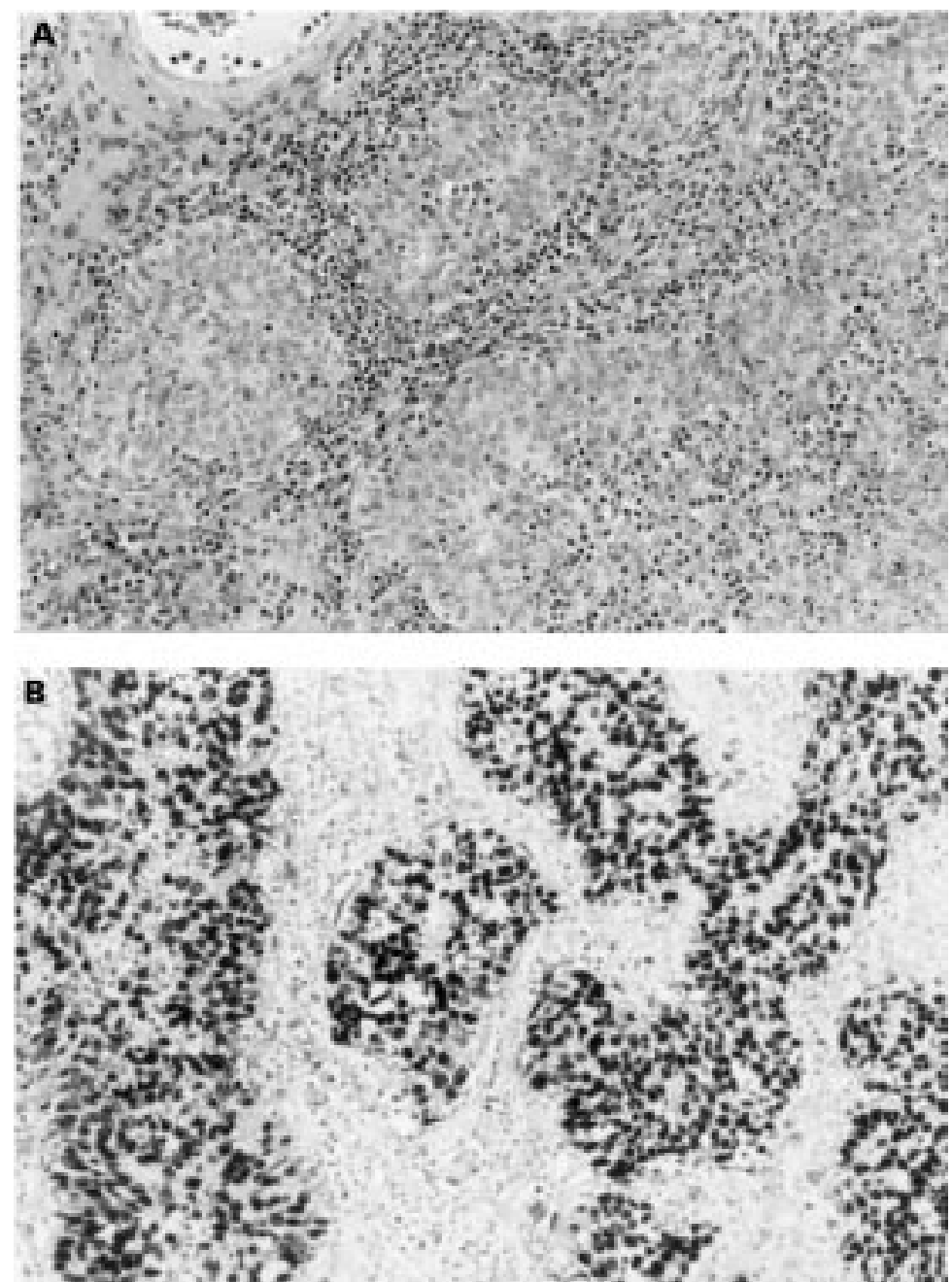

Figure 2 Case 1. (A) Non-keratinising carcinoma of nasopharyngeal type in the middle ear. Trabeculae of malignant cells with pavement arrangement of the basal layers are present. The nuclei are monotonous, pale, and vesicular with prominent nucleoli. Dense lymphoid infiltrate is present in the stroma and squamous epithelium of the ear canal is seen in the upper field. Haematoxylin and eosin. (B) The nuclei of the malignant cells are strongly positive for EBER. (EBER in situ hybridisation; digoxigenin labelled probe.)

of the nasopharynx. The clinical presentation included otitis media and aural polyp in five cases, impaired hearing and facial nerve palsy in one case, and a mass behind the ear involving the mastoid bone in one case.

PROCEDURES

In situ hybridisation for EBV encoded RNAs (EBER) using a digoxigenin labelled riboprobe was performed as described previously. ${ }^{1}$ Control hybridisation using a ${ }^{35}$ S-labelled antisense riboprobe spanning nucleotide 801-1131 of the human $\beta$ actin cDNA was performed to confirm the RNA integrity as described. ${ }^{2}$ Immunohistochemical staining for EBV latent membrane protein-1 (LMP-1) (CS1-4, 1:50, Dako, Copenhagen, Denmark) and EBNA2 (PE2, 1:50, Dako) were performed using the standard streptavidin-biotin-peroxidase method for the EBER positive cases. The sections were pretreated for antigen retrieval by microwave $\left(95^{\circ} \mathrm{C}\right.$ in citrate buffer $\mathrm{pH} 6.0$ for 30 minutes). Cell blocks prepared from formalin fixed Raji and B95-8 cell lines harbouring 50 copies and lytic form of EBV were used as positive control.

\section{Results}

One of the five non-keratinising squamous cell carcinomas and one of the two undifferentiated carcinomas were positive for EBER. Both EBER positive cases showed the morphology of lymphoepithelioma, with abundant lymphoid cells in the stroma. They were both negative for LMP-1 and EBNA2. The clinical and pathological features of the two cases are illustrated as follows:

\section{CASE 1}

This 37 year old Filipino woman presented with a right facial nerve palsy and impaired hearing for one year. She complained of recent increasing pain in the right temporal scalp region. There were no nasal symptoms. Examination with an otoscope showed granulation in the right external ear canal. She also had an enlarged left upper cervical lymph node. Radiography of the mastoid bone showed sclerotic changes. Computed tomography of the base of skull, nasopharynx, and neck was performed. There was bony destruction of the right petrous temporal bone. A large tumour was noted arising from the petrous bone, with intracranial extension into the right middle cranial fossa (fig 1). The nasopharynx was not involved. A right temporal craniotomy was performed with the intracranial portion of tumour removed and sent for histological examination. The tumour was that of a non-keratinising carcinoma of nasopharyngeal type. The malignant cells were arranged in trabeculae with pavement arrangement of the basal layers. The nuclei were monotonous, pale, and vesicular with prominent nucleoli. There was abundant lymphoid infiltrate in the stroma (fig 2A). In situ hybridisation showed strong expression of EBER in the malignant cells (fig 2B).

In view of the strong resemblance of the tumour to those arising from the nasopharynx, repeated examination of the nasopharynx by indirect or flexible nasopharyngoscopy was performed but no lesion was seen. Multiple random biopsies of the nasopharynx did not reveal any malignancy on three occasions. Fine needle aspiration of the left cervical lymph node did not yield adequate material for diagnosis. The serum titre of IgA against EBV viral capsid antigen was less than 1:10.

After careful review of the clinical and pathological findings, this case was considered to be a primary non-keratinising carcinoma of nasopharyngeal type arising from the middle ear. The cause of the left cervical lymphadenopathy remains unknown.

The patient returned to Philippines after recovery from the craniotomy, and no further follow up data were available.

CASE 2

This 37 year old man presented with a history of left ear discharge, tinnitus, and impaired hearing for five years. On examination he had a left facial nerve, VI, and XII nerve palsy. Clinical examination revealed a polyp in the left external auditory canal. Computed tomography showed a soft tissue mass in the left middle 

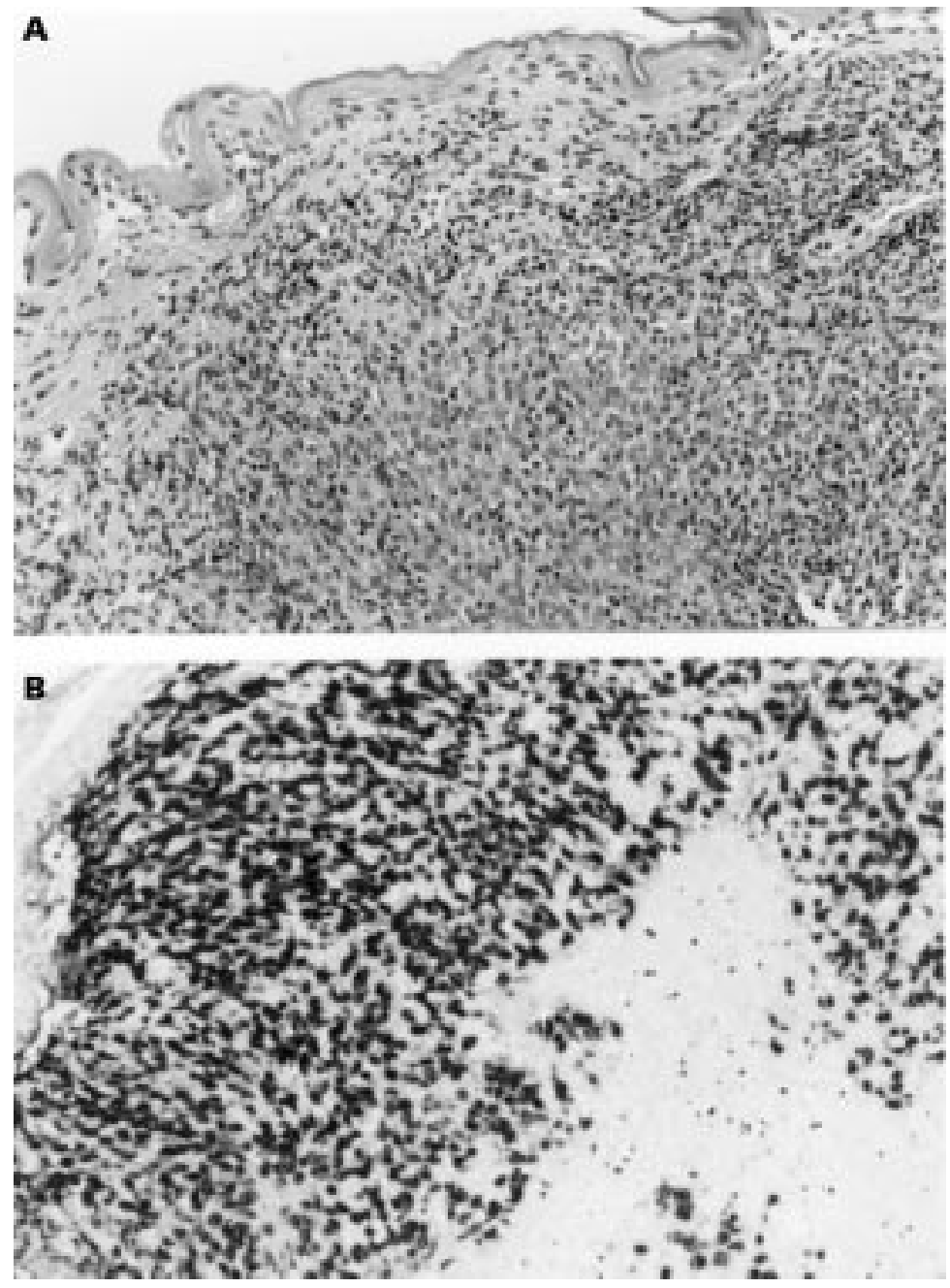

Figure 3 Case 2. (A) Undifferentiated carcinoma of nasopharyngeal type in the middle ear. Syncytial sheets of malignant cells in a dense lymphoid stroma with the ear canal lining epithelium present in the upper field. (B) Strong expression of EBER in the nuclei of the malignant cells. (EBER in situ hybridisation; digoxigenin labelled probe.)

ear cavity and external auditory canal with erosion of left petrous bone, left carotid canal, and left side of the body of sphenoid. Biopsy from the left ear showed an undifferentiated carcinoma, with the morphology of a lymphoepithelioma (fig 3). There were syncytial sheets of malignant cells with vesicular nuclei, prominent nucleoli, and abundant lymphoid infiltrate in the stroma. Multiple site biopsy of the nasopharynx was performed and was normal. The serum titre of IgA against EBV viral capsid antigen was less than $1: 10$.

He was treated with radiotherapy to the left middle ear and the surrounding areas. The tumour did not resolved with radiotherapy. $\mathrm{He}$ subsequently developed progressive disease, and was treated with multiple courses of chemotherapy and palliative radiotherapy. $\mathrm{He}$ was alive with disease 29 months after initial diagnosis.

\section{Discussion}

Although carcinoma of the ear is very rare, and most are keratinising squamous cell carcinomas, these cases show that tumours with identical morphology to those arising from the nasopharynx do exist in the middle ear and they can also harbour the Epstein-Barr virus. As in other patients with carcinoma of the ear, one of these patients (case 2) also had a long history of otitis media, with ear discharge and hearing problems. Both patients presented with very advanced stage disease. The question why EBV associated carcinoma is so common in the nasopharynx yet rare in the middle ear cavity, despite similar embryonic origin, remains unknown.

Although EBV associated carcinomas are uncommon outside the nasopharynx, they have been reported in diverse anatomical locations such as the salivary glands, ${ }^{34}$ nose and paranasal sinuses, ${ }^{15}$ tonsil ${ }^{6}{ }^{6}$ lacrimal sac, ${ }^{7}$ thymus, ${ }^{89}$ lung, ${ }^{10-12}$ stomach, ${ }^{13-15}$ and bile duct. ${ }^{16}$ In this study, we have documented the middle ear as another possible site for such tumours. In particular, these patients may have a long history of otitis media which may thus mask the presence of a tumour. Awareness of this information has important clinical and therapeutic implications, especially in the management of patients who presented with EBV positive cervical metastases with unknown primary.

The viral protein LMP-1, with transforming properties in vitro, was not expressed in the two EBV associated carcinomas in this series. In fact, the expression of LMP-1 in EBV associated carcinomas varies in different anatomical sites. It is expressed in most of salivary gland lymphoepithelial carcinomas, ${ }^{47} \quad 44 \%$ of lymphoepithelioma-like carcinomas in the lung, ${ }^{12} 29 \%$ of EBV associated sinonasal carcinomas, ${ }^{1}$ but not in EBV associated gastric carcinomas. ${ }^{18}$ In nasopharyngeal carcinoma, although LMP-1 is detected in variable proportions, ranging from $22 \%$ to $78 \%,{ }^{19} 20$ one study reported that all tumour cells in the preinvasive phase expressed LMP-1. ${ }^{21}$ Thus we cannot exclude the possibility that there may be transient expression of LMP-1 protein in certain stages of development of these tumours. 1 Leung SY, Yuen ST, Chung LP, et al. Epstein-Barr virus is

2 Leung SY, Wong MP, Chung LP, et al. Monocyte chemoattractant protein-1 expression and macrophage infiltration tractant protein-1 expression and macrophage in
in gliomas. Acta Neuropathol Berl 1997;93:518-27.

3 Hamilton-Dutoit SJ, Hamilton TM, Nielsen NH, et al. Undifferentiated carcinoma of the salivary gland in Greenlandic Eskimos: demonstration of Epstein-Barr virus DNA by in-situ nucleic acid hybridization. Hum Pathol 1991;22: $811-15$

4 Leung SY, Chung LP, Yuen ST, et al. Lymphoepithelial carcinoma of the salivary gland: in situ detection of
Epstein-Barr virus. $\mathcal{F}$ Clin Pathol 1995;48:1022-7.

5 Lopategui JR, Gaffey MJ, Frierson HF, et al. Detection of Epstein-Barr viral RNA in sinonasal undifferentiated carcinoma from Western and Asian patients. Am 7 Surg Pathol noma from West

6 Nicholls JM, Pittaluga S, Chung LP, et al. The association between carcinoma of the tonsil and Epstein-Barr virus-a study using radiolabelled in situ hybridization. Pathology 1994;26:94-8.

7 Leung SY, Chung LP, Ho CM, et al. An Epstein-Barr virus positive undifferentiated carcinoma in the lacrimal sac. Histopathology 1996;28:71-5.

8 Leyvraz S, Henle W, Chahinian AP, et al. Association of Epstein-Barr virus with thymic carcinoma. $N$ Engl f Med 1985;312:1296-9.

9 Dimery IW, Lee JS, Blick M, et al. Association of the Epstein-Barr virus with lymphoepithelioma of the thymus. Cancer 1988;61:2475-80.

10 Begin LR, Eskandari J, Joncas J, et al. Epstein-Barr virus related lymphoepithelioma-like carcinoma of lung. F Surg ol 1987;36:280-3.

11 Butler AE, Colby TV, Weiss L, et al. Lymphoepithelioma-like carcinoma of the lung. Am f Surg Pathol 1989;13:632-9. 
12 Wong MP, Chung LP, Yuen ST, et al. In situ detection of Epstein-Barr virus in non-small cell lung carcinomas. $\mathcal{F}$ Epstein-Barr virus in n

13 Shibata D, Tokunaga M, Uemura Y, et al. Association of Epstein-Barr virus with undifferentiated gastric carcinomas with intense lymphoid infiltration. Lymphoepitheliomalike carcinoma. Am f Pathol 1991;139:469-74.

14 Shibata D, Weiss LM. Epstein-Barr virus-associated gastric adenocarcinoma. Am f Pathol 1992;140:769-74.

15 Yuen ST, Chung LP, Leung SY, et al. In situ detection of Epstein-Barr virus in gastric and colorectal adenocarcinomas. Am 7 Surg Pathol 1994;18:1158-63.

16 Hsu HC, Chen CC, Huang GT, et al. Clonal Epstein-Barr virus associated cholangiocarcinoma with
lymphoepithelioma-like component. Hum Pathol 1996;27: 848-50.

17 Wen S, Mizugaki Y, Shinozaki F, et al. Epstein-Barr virus (EBV) infection in salivary gland tumors: lytic EBV infec- tion in nonmalignant epithelial cells surrounded by EBV-positive T-lymphoma cells. Virology 1997;227:484-7. 18 Imai S, Koizumi S, Sugiura M, et al. Gastric carcinoma: monoclonal epithelial malignant cells expressing EpsteinBarr virus latent infection protein. Proc Natl Acad Sci USA 1994;91:9131-5.

19 Niedobitek G, Young LS, Sam CK, et al. Expression of Epstein-Barr virus genes and of lymphocyte activation molecules in undifferentiated nasopharyngeal carcinomas. Am F Pathol 1992;140:879-87.

20 Stewart JP, Arrand JR. Expression of the Epstein-Barr virus latent membrane protein in nasopharyngeal carcinoma biopsy specimens. Hum Pathol 1993;24:239-42.

21 Pathmanathan R, Prasad U, Sadler R, et al. Clonal proliferations of cells infected with Epstein-Barr virus in preinvasive lesions related to nasopharyngeal carcinoma. $N$ Engl F Med 1995;333:693-8. 